ESAIM: COCV 21 (2015) 857-875

DOI: $10.1051 / \mathrm{cocv} / 2014053$
ESAIM: Control, Optimisation and Calculus of Variations

www.esaim-cocv.org

\title{
ON THE PROPERNESS OF AN IMPULSIVE CONTROL EXTENSION OF DYNAMIC OPTIMIZATION PROBLEMS
}

\author{
D.Y. Karamzin ${ }^{1,2}$, V.A. De Oliveira ${ }^{1}$, F.L. Pereira ${ }^{3}$ And G.N. Silva ${ }^{1}$
}

\begin{abstract}
This article investigates the properness, or well-posedness, of impulsive extension of a conventional optimal control problem. This includes both well-posedness of the solution to impulsive control systems arising as result of an impulsive extension of ordinary differential systems, and existence theorems. Well-posedness in the classic Cauchy sense is proved. Approximation lemmas that guarantee sensitivity to small perturbations in control variables are obtained. Filippov type existence theorems are established. A model example is provided to show the relevance of the impulsive controls problems which are under study.
\end{abstract}

Mathematics Subject Classification. 49N25,49J15.

Received October 27, 2013. Revised June 16, 2014.

Published online May 20, 2015.

\section{INTRODUCTION}

Some optimal control problems do not have solution in the class of classic controls. This suggests the need for a relaxation or extension of the control problem ensuring the existence of a solution in some enlarged class of controls, permitting admissible arcs to have jumps.

A proper, or well-posed, extension procedure includes the following steps:

1. Extension of the class of controls. Definition of the control in the extended problem as element of a certain metric space. The concept of "proximity" for controls.

2. Extension of the class of trajectories. Definition of the trajectory in the extended problem as a solution to the extended dynamical system. The well-posedness of the solution consists in verifying that "close" controls yield "close" trajectories.

3. Existence theorems. These are conditions under which the proposed extension procedure is proper or successful in the sense that the extended problem will have a solution.

As usual, the above procedure should also preserve the rule: "the infimum costs for the original and the extended problem are the same". This plan is unfolded in Sections 2-5.

\footnotetext{
Keywords and phrases. Optimal control extensions, well-posedness of solutions, existence of solutions, impulsive control.

1 UNESP - Universidade Estadual Paulista, Department of Applied Mathematics, São José do Rio Preto, SP, Brazil. dmitry_karamzin@mail.ru, antunes@ibilce.unesp.br \& gsilva@ibilce.unesp.br

2 Computing Center of the Russian Academy of Sciences, Moscow, Russia.

3 SYSTEC, Faculdade de Engenharia, Universidade do Porto, Rua Dr. Roberto Frias, s/n, 4200-465 Porto, Portugal.

flp@fe.up.pt
} 


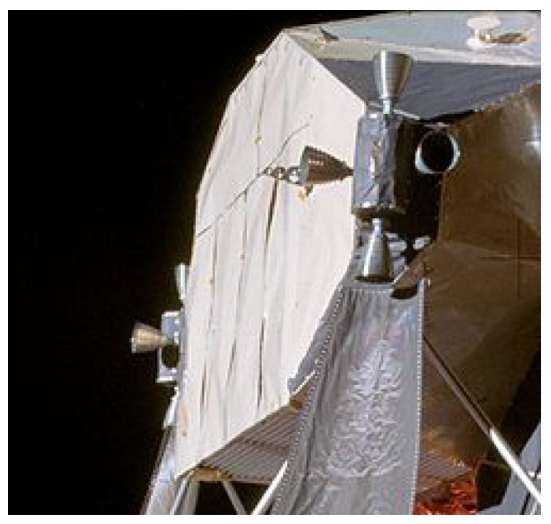

Figure 1. RCT blocks on the Apollo Lunar Module.

(Source: http://www.hq.nasa.gov/office/pao/History/alsj/a14/AS14-66-9254HR.jpg).

Impulsive control systems, in its various guises including existence of solution, well-posedness of solution, sensitivity analysis, etc., have been investigated by various researchers. It is relevant to mention the works by Aronna and Rampazzo [1], Arutyunov, Dykhta, Jacimovic and Pereira [4,5], Bressan and Rampazzo [11,12], Code and Loewen [13], Code and Silva [14], Dykhta and Samsonyuk [15], Forcadel, Rao and Zidani [16], Kurzhanskii and Daryin [17], Miller and Rubinovich [19], Pereira and Silva [23], Pereira, Silva and de Oliveira [24], Rishel [30], Silva and Vinter [26, 27], Vinter and Pereira [29], Warga [31], Wolenski and Zabic [32,33], among others. The concept of impulsive control considered in this work is being developed along the lines of [7-9]. The innovative matter of this approach is related to the issue of controlling system jumps raised and discussed in [9].

Impulsive controls are widely used in various engineering applications, in particular space navigation where one of the goals has generally been to minimize fuel consumption [18]. Impulsive controls, then, are helpful in the analysis since they provide mathematically precise solutions to the extended problem in which the fuel consumption rate is allowed to take unbounded values. The latter assumption is quite realistic because the process of fuel consumption in jet propulsions has an explosive character. That is, when a considerable quantity of fuel is consumed within short time intervals and, therefore, the value of the consumption rate can be very high. The consideration of impulsive controls simplifies the mathematical analysis of the problem, thus, making the necessary optimality conditions easier for application.

In this work, we investigate impulsive control processes of a specific nature. Their main feature is that the state trajectory can be controlled while the impulse develops. This means that there are conventional type controls that enable to steer the trajectory "inside" of the system's jump. Examples of such processes can be found in problems related to aerospace flights where the mass of a flying object may change while the impulse occurs, due to the fuel consumption. Since the mass changes, the center of gravity also changes. However, this quick alteration in the parameters of the system requires correction in control during the time of the impulse. In this way, the conventional control affects the jump. The changing of the center of gravity or of the mass distribution can also disturb the rotation motion of the object. For example, an unwanted rotation motion may emerge. Then, it is important to know the right control mode for the new mass parameters in order to save fuel and, at the same time, to keep the flying object with the non-perturbed rotation motion. In other words, it is important to provide a correct joint control for both modes motion: translation and orientation.

In aerospace navigation, situations in which it is necessary to jointly control multiple force lines are numerous. Examples include the operation of a lunar module (landing), shuttle docking, satellite reorientation, and others. Apart from powerful jet propulsions, these spacecrafts have also small jet-engines called thrusters. The function of the thrusters is to steer the spacecraft, control the attitude, or doing some other precise and intricate maneuver such as docking or even landing. For example, the Apollo Lunar Module has RCT - Reaction Control Thrusters - (see Fig. 1). By using these thrusters, the Lunar Module can maneuver over the lunar surface during landing. 


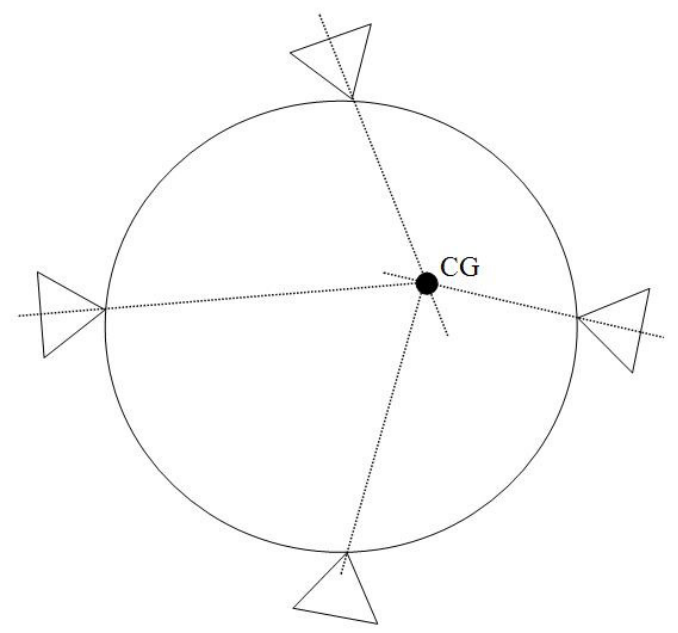

Figure 2. Force lines passing through the CG.

The thrusters are set sidewards generating the two above mentioned modes: translation mode (when thrusters are used to alter the spacecraft velocity) and orientation mode (when thrusters are used to rotate and orient the spacecraft). In what concerns the translation mode, the thrusters have one important requirement: the line of the force provided by the translation thrusters should always intersect the spacecraft center of gravity as shown in Figure 2. Otherwise an unwanted rotation and/or waste of fuel results. However, even when all the line forces pass through the center of gravity, the perturbation of the rotation motion is unavoidable whenever the angular velocity is not zero. In order to notice this, we need to take into account the non-zero angular momentum of the exhaust. Note that the same thrusters have to provide translation and orientation of the spacecraft simultaneously.

In Section 6, the analysis of a model example in relation to the problem of the automated control by multiple thrusters (in a reduced form) is presented. This example motivates the research work in this article.

\section{Problem Statement}

Consider the following impulsive control problem.

$$
\begin{cases}\text { Minimize } & c(p) \\ \text { subject to } & \mathrm{d} x=f(x, u, t) \mathrm{d} t+g(x, u, t) \mathrm{d} \vartheta, \\ & \mathrm{d} y=d|\vartheta|, y\left(t_{0}\right)=0, \\ & \varphi(x, t) \leq 0 \\ & u(t) \in U, \text { range }(\vartheta) \subset K, \\ & p \in S, t \in T,\end{cases}
$$

which is an impulsive extension of conventional optimal control problems, see $[2,21,25,28]$.

Above, the functions $c: \mathbb{R}^{2 n+1} \rightarrow \mathbb{R}^{1}, f: \mathbb{R}^{n} \times \mathbb{R}^{m} \times \mathbb{R}^{1} \rightarrow \mathbb{R}^{n}, g: \mathbb{R}^{n} \times \mathbb{R}^{m} \times \mathbb{R}^{1} \rightarrow \mathbb{R}^{n \times k}, \varphi: \mathbb{R}^{n} \times \mathbb{R}^{1} \rightarrow \mathbb{R}^{l}$ are continuous, $T=\left[t_{0}, t_{1}\right]$ is a fixed time interval, $p=\left(x_{0}, x_{1}, y_{1}\right)$, where $x_{0}=x\left(t_{0}\right), x_{1}=x\left(t_{1}\right)$ and $y_{1}=y\left(t_{1}\right)$, is the so called endpoint vector, $S$ is a closed set in $\mathbb{R}^{2 n+1}, U$ is a closed set in $\mathbb{R}^{m}, K$ is a closed convex cone in $\mathbb{R}^{k}, c(p)$ is the cost function to be minimized, the vector-function $\varphi$ defines the state constraints, and $\vartheta$ is the impulsive control, see [9]. The measure $|\vartheta|$ designates the variation measure of the impulsive control, whereas $y_{1}$ becomes its total variation.

Recall the impulsive control concept from [9]. Let $K$ be a nonempty closed convex cone in $\mathbb{R}^{k}$. Consider a Borel vector measure $\mu$ such that range $(\mu) \subset K$. Let $V(\mu)$ be the set of scalar-valued nonnegative Borel measures $\nu$ 
such that $\exists \mu_{i}$ satisfying range $\left(\mu_{i}\right) \subset K$ and $\left(\mu_{i},\left|\mu_{i}\right|\right) \stackrel{*}{\rightarrow}(\mu, \nu)$. Here, the symbol $\stackrel{*}{\rightarrow}$ means weak*-convergence, i.e., all the coordinates of $\mu_{i}$ converge in $C^{*}(T)$ endowed with its weak star topology, and also the variation measure $\left|\mu_{i}\right|$ converges weakly* to $\nu$, where $C^{*}(T)$ is the dual space to $C(T)$. For instance, if $K$ is contained in one of the orthants, then $V(\mu)=\{|\mu|\}$ (single-point set).

Consider an arbitrary scalar measure $\nu \in V(\mu)$ and a number $\tau \in T$. Let a measurable vector function $v_{\tau}:[0,1] \rightarrow K$ be such that

(i) $\sum_{j=1}^{k}\left|v_{\tau}^{j}(s)\right|=\nu(\tau)$, a.a. $s \in[0,1]$, and

(ii) $\int_{0}^{1} v_{\tau}^{j}(s) d s=\mu^{j}(\tau), j=1, \ldots, k$.

A family of vector functions $\left\{v_{\tau}\right\}$, depending on the real parameter $\tau \in T$, is said to be attached to the vector measure $(\mu, \nu)$, if, for every $\tau$, conditions (i) and (ii) above hold.

Definition 2.1. The impulsive control is a triple $\vartheta=\left(\mu,\left\{u_{\tau}, v_{\tau}\right\}\right)$, where $\left\{u_{\tau}\right\}$ is a family of measurable and uniformly bounded in $\tau$ vector functions defined on the segment $[0,1]$ with values in $\mathbb{R}^{m}$, if there exists $\nu \in V(\mu)$ such that $\left\{v_{\tau}\right\}$ is attached to $(\mu, \nu)$. The measure $\nu$ is called variation of the impulsive control $\vartheta$. We denote it by $|\vartheta|$.

The ordinary control function $u(\cdot)$ is assumed to be measurable and essentially bounded w.r.t. both Lebesgue $\ell$ and Lebesgue-Stieltjes $|\vartheta|$ measures.

Take an impulsive control $\vartheta=\left(\mu,\left\{u_{\tau}, v_{\tau}\right\}\right)$, a number $\tau \in T$ and a vector $a \in \mathbb{R}^{n}$. Denote by $x_{\tau}(\cdot)=x_{\tau}(\cdot, a)$ the solution to the following dynamical system

$$
\left\{\begin{array}{l}
\dot{x}_{\tau}(s)=g\left(x_{\tau}(s), u_{\tau}(s), \tau\right) v_{\tau}(s), s \in[0,1] \\
x_{\tau}(0)=a
\end{array}\right.
$$

The function of bounded variation $x(t)$ on the interval $T$ is called solution to the differential equation in (2.1), corresponding to the control $(u, \vartheta)$ and the starting value $x_{0}$, if $x\left(t_{0}\right)=x_{0}$ and, for every $t \in\left(t_{0}, t_{1}\right]$,

$$
x(t)=x_{0}+\int_{t_{0}}^{t} f(x, u, \tau) \mathrm{d} \tau+\int_{\left[t_{0}, t\right]} g(x, u, \tau) \mathrm{d} \mu_{\mathrm{c}}+\sum_{\tau \leq t}\left[x_{\tau}\left(1, x\left(\tau^{-}\right)\right)-x\left(\tau^{-}\right)\right],
$$

where $\mu_{c}$ designates the continuous component of $\mu$. Note, that the sum in (2.2) is well defined since there is only a countable set of points $\tau$, where $v_{\tau}(\cdot)$ is a nonzero function.

The state constraints $\varphi(x, t) \leq 0$ in $(2.1)$ are understood in a wider sense than that of a conventional inequality. This is due to the presence of the impulsive component. Let us make precise this meaning. Let us be given any admissible trajectory $x(\cdot)$ corresponding to the impulsive control $\vartheta$. Consider the variation measure $|\vartheta|$. Denote $\operatorname{Ds}(\vartheta):=\{\tau \in T:|\vartheta|(\tau)>0\}$ the set of atoms of $|\vartheta|$ (impulses). Now, the inequality $\varphi(x, t) \leq 0$ has to be understood in the following generalized sense:

$$
\varphi(x, t) \leq 0 \Leftrightarrow\left\{\begin{array}{l}
\varphi(x(t), t) \leq 0, \quad \text { a.a. } t \in T, \\
\varphi\left(x_{\tau}(s), \tau\right) \leq 0, \text { a.a. } s \in[0,1], \forall \tau \in \operatorname{Ds}(\vartheta) .
\end{array}\right.
$$

The collection $(x, y, u, \vartheta)$ is called a control process, if $\mathrm{d} y=d|\vartheta|$ and (2.2) holds. A control process is said to be admissible, if all the constraints of problem (2.1) are satisfied. An admissible process $(\hat{x}, \hat{y}, \hat{u}, \hat{\vartheta})$ is said to be optimal if, for any admissible process $(x, y, u, \vartheta)$, the inequality $c(\hat{p}) \leq c(p)$ holds, where $\hat{p}=\left(\hat{x}\left(t_{0}\right), \hat{x}\left(t_{1}\right), \hat{y}\left(t_{1}\right)\right)$. 


\section{Preliminaries}

This section includes auxiliary technical constructions needed for derivation of the main results. Consider the set of all controls in (2.1), that is all pairs $\wp=(u, \vartheta)$. Denote this set by $\mathcal{P}$.

Take a pair $\wp=(u, \vartheta)$, where $\vartheta=\left(\mu,\left\{u_{\tau}, v_{\tau}\right\}\right)$. Consider the function $\pi: T \rightarrow[0,1]$, defined by

$$
\pi(t)=\frac{t-t_{0}+\nu\left(\left[t_{0}, t\right]\right)}{c}, t \in\left(t_{0}, t_{1}\right], \pi\left(t_{0}\right)=0,
$$

where $\nu=|\vartheta|$ is the variation of the impulsive control (see [9]) and $c=t_{1}-t_{0}+\nu(T)$, which is also known as Lebesgue discontinuous time variable change. There exists the inverse function $\theta(s):[0,1] \rightarrow T$ such that:

(a) $\theta(s)$ is nondecreasing function on $[0,1]$;

(b) $\theta(s)$ is absolutely continuous, and satisfies $|\theta(s)-\theta(t)| \leq c|s-t| \forall s, t$;

(c) $\theta(s)=\tau \forall s \in \Gamma_{\tau}, \forall \tau \in T$, where $\Gamma_{\tau}=\left[\pi\left(\tau^{-}\right), \pi\left(\tau^{+}\right)\right]$.

Note that $\pi(t)$ maps $(\ell+|\vartheta|)$-measurable sets into $\ell$-measurable sets. Indeed, this follows directly from the definition of $\pi$ and also from the representation of a measurable set as a union of a Borel and a zero measure sets. Therefore, if the set $A$ is $(\ell+|\vartheta|)$-measurable, then $\theta^{-1}(A)$ is measurable. This implies measurability of the function $u(\theta(s))$ as soon as $u(t)$ is $(\ell+|\vartheta|)$-measurable. So, now, for example, the variable change

$$
\int_{t_{0}}^{t_{1}}|u(t)| \mathrm{d} \mu=c \int_{0}^{1}|u(\theta(s))| m(\theta(s)) \mathrm{d} s,
$$

where $m$ is Radon-Nikodym derivative of $\mu$ with respect to $\ell+|\vartheta|$, is well defined.

Let $\phi$ be any continuous function in its $\operatorname{arguments}(x, u, t)$, which take values in $\mathbb{R}^{l}$. Introduce the nonlinear operator

$$
\operatorname{Ext}[\phi, \vartheta](s):=\left\{\begin{array}{l}
\phi\left(x_{\tau}\left(\xi_{\tau}(s)\right), u_{\tau}\left(\xi_{\tau}(s)\right), \tau\right) \text { if } \exists \tau \in \operatorname{Ds}(|\vartheta|): s \in \Gamma_{\tau}, \\
\phi(x(\theta(s)), u(\theta(s)), \theta(s)) \text { otherwise }
\end{array}\right.
$$

mapping $[0,1]$ in $\mathbb{R}^{l}$. Here,

$$
\xi_{\tau}(s)=\frac{s-\pi\left(\tau^{-}\right)}{\ell\left(\Gamma_{\tau}\right)}: \Gamma_{\tau} \rightarrow[0,1]
$$

The set $\mathcal{P}$ can be endowed with a metric as follows. Consider the distribution function $F(t, \nu)$ related to the scalar Borel measure $\nu=|\vartheta|$, i.e., $F(t, \nu)=\nu\left(\left[t_{0}, t\right]\right), t \in\left(t_{0}, t_{1}\right]$, with $F\left(t_{0}, \nu\right)=0$. Take two elements $\wp_{1}$, and $\wp_{2}$ of $\mathcal{P}$, and let $\wp_{1}=\left(u_{1}, \vartheta_{1}\right), \wp_{2}=\left(u_{2}, \vartheta_{2}\right)$. Let also $\zeta_{j}$ be the solution to the simplest control system $d \zeta_{j}=\mathrm{d} \vartheta_{j}, \zeta_{j}\left(t_{0}\right)=0, j=1,2$.

Let us define the distance in $\mathcal{P}$ by the formula

$$
\begin{aligned}
\rho\left(\wp_{1}, \wp_{2}\right)=\left|\nu_{1}(T)-\nu_{2}(T)\right| & +\int_{t_{0}}^{t_{1}}\left|F\left(t, \nu_{1}\right)-F\left(t, \nu_{2}\right)\right| \mathrm{d} t+\max _{s \in[0,1]}\left|\operatorname{Ext}\left[\zeta_{1}(\cdot), \vartheta_{1}\right](s)-\operatorname{Ext}\left[\zeta_{2}(\cdot), \vartheta_{2}\right](s)\right| \\
& +\int_{0}^{1}\left|\operatorname{Ext}\left[u_{1}(\cdot), \vartheta_{1}\right](s)-\operatorname{Ext}\left[u_{2}(\cdot), \vartheta_{2}\right](s)\right| \mathrm{d} s .
\end{aligned}
$$

The functions $\operatorname{Ext}\left[\zeta_{j}(\cdot), \vartheta_{j}\right]$ are continuous. The functions $\operatorname{Ext}\left[u_{j}(\cdot), \vartheta_{j}\right]$ are measurable and essentially bounded, as it was mentioned above. Therefore, the maximum and the integral in (3.2) are defined properly. It is easy to see that the function $\rho(\cdot, \cdot)$ satisfies all the axioms of a metric. Thus, $\mathcal{P}$ is a metric space. This space is not complete. Being the functions $u$ considered as functions of $L_{1}$ instead of $L_{\infty}$, the space $\mathcal{P}$ would be complete. Designate the convergence in $\mathcal{P}$ by $\stackrel{\rho}{\rightarrow}$. Note that any element $(u, v)$, where $v(t) \in K$, can be treated as element $(u, \vartheta)$ of the space $\mathcal{P}$, if we put $\vartheta=(\mu ;\{0,0\})$, with $d \mu=v(t) \mathrm{d} t$. 
The idea of considering metric (3.2) is clearly revealed by the following two statements:

(A) The set of usual bounded controls $(u, v)$ is everywhere dense in $\mathcal{P}$.

(B) Passing to the limit in the nonlinear impulsive control system w.r.t. $\rho$ is correct.

While the property $(\mathrm{A})$ is rather clear (it can be proved by the mere construction of the required sequence of ordinary controls $\left(u_{i}, v_{i}\right)$, the details are omitted), the property (B) requires a more precise formulation and proof. This is the role of Lemma 4.2 from the next section.

\section{WELL-POSEDNESS OF SOLUTION}

Consider the control differential system:

$$
\mathrm{d} x=f(x, u, t) \mathrm{d} t+g(x, u, t) \mathrm{d} \vartheta, \quad t \in T=\left[t_{0}, t_{1}\right],
$$

which includes an ordinary measurable essentially bounded control $u$ and an impulsive control $\vartheta=\left(\mu,\left\{u_{\tau}, v_{\tau}\right\}\right)$. The definition of impulsive control as well as definition of solution $x(\cdot)$ to impulsive system (4.1) was given in Section 2. Below, the well-posedness of this solution (a) in the classical Cauchy sense and (b) with respect to the metric (3.2) is established. Recall that the mappings $f$ and $g$ are continuous, and the set $U$ is closed.

\subsection{Well-posedness in the Cauchy sense}

The following lemma is a simple generalization of the classical results.

Lemma 4.1. Let $x_{0} \in \mathbb{R}^{n}$ and $(u, \vartheta) \in \mathcal{P}$. Suppose $m(t): T \rightarrow \mathbb{R}^{1}$ is an integrable function such that:

$$
|f(x, u, t)|+|g(x, u, t)| \leq m(t)(1+|x|) \quad \forall x, u, t \in \mathbb{R}^{n} \times U \times T .
$$

Then, on the interval $T$, there exists a solution $x(\cdot)$ to $(4.1)$, corresponding to the control $(u, \vartheta) \in \mathcal{P}$ and to the starting value $x\left(t_{0}\right)=x_{0}$.

In case (4.2) does not hold true, but $f, g$ are continuously differentiable in $x$, and $t_{0} \notin \mathrm{Ds}(|\vartheta|)$, there exists a number $\delta>0$ such that the solution $x(\cdot)$ exists only locally on the interval $\left[t_{0}, t_{0}+\delta\right]$.

In both cases the solution is unique.

Moreover, small perturbations of the starting value $x_{0}$ produce small deviations of the solution

$$
\left|x(t)-x^{\prime}(t)\right| \leq \text { const }\left|x_{0}-x_{0}^{\prime}\right| \forall t \in T,
$$

where $x^{\prime}(t)$ is the solution to (4.1) corresponding to the starting value $x_{0}^{\prime}$ and the same control $(u, \vartheta) \in \mathcal{P}$.

Proof. The proof is simple and based on the Lebesgue discontinuous change of time variable. Take $(u, \vartheta) \in \mathcal{P}$. Consider the function $\pi(t)$ from (3.1) and its inverse function $\theta(s):[0,1] \rightarrow T$. It is not restrictive to assume that $c=1$ in (3.1).

Let $\tilde{u}(s)=\operatorname{Ext}[u, \vartheta](s)$. The function $\tilde{u}$ is measurable. Let us put

$$
\begin{aligned}
& \alpha(s)=\left\{\begin{array}{l}
m_{1}(\theta(s)), \text { if } s \notin \bigcup_{\tau \in \operatorname{Ds}(\vartheta)} \Gamma_{\tau}, \\
0, \quad \text { otherwise, }
\end{array}\right. \\
& v(s)= \begin{cases}\frac{m_{2}(\theta(s))}{(1-\alpha(s))}, & \text { if } s \notin \bigcup \Gamma_{\tau} \text { and } \alpha(s)<1, \\
\ell^{-1}\left(\Gamma_{\tau}\right) v_{\tau}\left(\xi_{\tau}(s)\right), & \text { if } s \in \Gamma_{\tau},\end{cases}
\end{aligned}
$$

where, $m_{1}$ and $m_{2}$ are the Radon-Nikodym derivatives of the measures $\ell$ and $\mu_{c}$ with respect to $\ell+|\vartheta|$, respectively, and $\xi_{\tau}(s)$ was introduced above. If $\alpha(s)=1$, the values of $v(s)$ may be taken as arbitrary unit vectors from $K$, since the trajectory $x(\cdot)$ does not depend on those values. 
Note that $v(s) \in K$ and $|v(s)|=1$ due to $m_{1}(t)+\left|m_{2}(t)\right|=1$ a.a.t $\notin \operatorname{Ds}(\vartheta)$ w.r.t. $\ell+|\vartheta|$ measure. Consider now the auxiliary system

$$
\left\{\begin{array}{l}
\dot{y}(s)=\alpha(s) f(y(s), \tilde{u}(s), \theta(s))+(1-\alpha(s)) g(y(s), \tilde{u}(s), \theta(s)) v(s), \\
y(0)=x_{0}
\end{array}\right.
$$

where $s \in[0,1]$. The system (4.4) is an ordinary differential system with measurable in $s$ right-hand. Therefore, due to the assumptions on $f$ and $g$ imposed in the lemma, (4.4) has (local or global) unique solution $y(\cdot)$. By performing now the discontinuous variable change $s=\pi(t)$ in (4.4) and by transforming (we omit the details which are straightforward), we conclude that trajectory $t \mapsto y(\pi(t))$ satisfies the solution concept from the Section 2. So, $x(t)=y(\pi(t)) \forall t$ and, moreover,

$$
\operatorname{Ext}[x, \vartheta](s)=y(s) .
$$

From the above arguments, it obviously follows the sensitivity to small perturbations in $x_{0}$. Indeed, due to Gronwall's inequality, from (4.4), one has

$$
\left|\operatorname{Ext}[x, \vartheta](s)-\operatorname{Ext}\left[x^{\prime}, \vartheta\right](s)\right| \leq \mathrm{const}\left|x_{0}-x_{0}^{\prime}\right| .
$$

Then, by definition, the estimate (4.3) holds.

This concludes the proof.

Note, that, additionally, by assuming Lipschitz continuity in $u$ we get a similar estimate and sensitivity to small perturbations in $\mathbb{L}_{1}$-norm of the ordinary control:

$$
\left|x(t)-x^{\prime}(t)\right| \leq \operatorname{const}\left(\left|x_{0}-x_{0}^{\prime}\right|+\left\|u-u^{\prime}\right\|_{\mathbb{L}_{1}}\right) \forall t \in T .
$$

However, the (4.3)-like estimate fails as soon as the impulsive control $\vartheta$ is perturbed. Let us proceed to the study of correctness with respect to perturbations in impulsive control variable.

\subsection{Well-posedness of passing to the limit}

So far, the well-posedness in the Cauchy sense for impulsive control systems has been established. Now, let us see the possibility of the well-posedness property when passing to limits in nonlinear impulsive control systems of the type (4.1).

Lemma 4.2. Let $\left(u_{i}, \vartheta_{i}\right) \stackrel{\rho}{\rightarrow}(u, \vartheta)$, where $\left(u_{i}, \vartheta_{i}\right),(u, \vartheta)$ are from $\mathcal{P}$. Assume that $x_{0, i} \rightarrow x_{0} \in \mathbb{R}^{n}$ and $\operatorname{Ext}\left[u_{i}, \vartheta_{i}\right](s)$ are uniformly bounded. Assume (4.2) be in force. Denote by $x_{i}(\cdot)$ the solution to (4.1), corresponding to the control $\left(u_{i}, \vartheta_{i}\right)$ and the starting value $x_{0, i}$, which by means of Lemma 4.1 exists on $T \forall i$.

Then, $\operatorname{Ext}\left[x_{i}, \vartheta_{i}\right](s) \rightrightarrows \operatorname{Ext}[x, \vartheta](s)$ uniformly in $s \in[0,1]$, where $x(\cdot)$ is the solution to (4.1), corresponding to the control $(u, \vartheta) \in \mathcal{P}$ and the starting value $x\left(t_{0}\right)=x_{0}$.

The latter implies

$$
x_{i}(t) \rightarrow x(t) \forall t \in(T \backslash \operatorname{Ds}(|\vartheta|)) \cup\left\{t_{0}, t_{1}\right\} .
$$

Proof. The proof is based on the discontinuous time variable change. It is not restrictive to assume that $f(\cdot, \cdot, t)=$ $0 \forall t \in D$, where $D$ is a set of zero measure on which the singular component of $|\hat{\vartheta}|$ has full measure. Since $\ell(D)=0$, the values of $f$ on $D$ are not involved in the construction of trajectory. We proceed similarly with values of $f$ in case of measures $\left|\vartheta_{i}\right|, i=1,2, \ldots$ Now $f(\cdot, \cdot, t)$ is measurable w.r.t. $\ell+|\vartheta|$ and $\left|\vartheta_{i}\right| \forall i$. This fact will be used below.

Consider the discontinuous time variable change $\pi(\cdot)$, see (3.1), related to the given impulsive control $\vartheta$ and designate its inverse function by $\theta:[0,1] \rightarrow T$. Consider the function

$$
m_{1}(t)=c \cdot \frac{\mathrm{d} \ell}{\mathrm{d}(\ell+|\vartheta|)}, \quad c=t_{1}-t_{0}+|\vartheta|(T),
$$


which is the Radon-Nikodym derivative of $\ell$ w.r.t. $\ell+|\vartheta|$ multiplied by the constant $c$. Here, $\ell$ is the usual Lebesgue measure. The function $m_{1}(t)$ is $(\ell+|\vartheta|)$-measurable.

We have, by definition, the following formula for $\theta(s)$ :

$$
\theta(s)=t_{0}+\int_{0}^{s} \alpha(\varsigma) \mathrm{d} \varsigma,
$$

where

$$
\alpha(\varsigma)= \begin{cases}m_{1}(\theta(\varsigma)), & \text { if } \varsigma \notin \bigcup_{\tau \in \operatorname{Ds}(|\vartheta|)} \Gamma_{\tau}, \\ 0, & \text { otherwise. }\end{cases}
$$

Indeed, when the measure $|\vartheta|$ is atomless, formula (4.5) is clear, since:

$$
t-t_{0}=\int_{t_{0}}^{t} \mathrm{~d} \varsigma=\int_{t_{0}}^{t} m_{1}(\varsigma) \mathrm{d} \pi(\tau)=\int_{0}^{\pi(t)} m_{1}(\theta(\varsigma)) \mathrm{d} \varsigma .
$$

When $|\vartheta|$ has a finite number of atoms $\tau_{1}, \ldots, \tau_{N}$, formula (4.5) is obtained by consecutive applications of the continuous time variable change on the segments $\left[\tau_{i}, \tau_{i+1}\right]$. Finally, for an arbitrary measure $|\vartheta|$, formula $(4.5)$ also holds because the measure $|\vartheta|$ can be approximated by measures with finite number of atoms.

Let

$$
m_{2}(t)=c \cdot \frac{\mathrm{d} \mu_{\mathrm{c}}}{\mathrm{d}(\ell+|\vartheta|)}
$$

be the Radon-Nikodym derivative of $\mu_{\mathrm{c}}$ w.r.t. $\ell+|\vartheta|$ multiplied by the constant $c$. The function $m_{2}(t)$ is $(\ell+|\vartheta|)$-measurable and essentially bounded.

Let us define the function

$$
\beta(\varsigma)= \begin{cases}c \cdot\left(\ell\left(\Gamma_{\tau}\right)\right)^{-1} v_{\tau}\left(\xi_{\tau}(\varsigma)\right), & \text { if } \exists \tau \in \operatorname{Ds}(|\vartheta|): \varsigma \in \Gamma_{\tau}, \\ m_{2}(\theta(\varsigma)), & \text { otherwise. }\end{cases}
$$

It is easy to see that

$$
\operatorname{Ext}[\zeta, \vartheta](s)=\int_{0}^{s} \beta(\varsigma) \mathrm{d} \varsigma, \text { where } \mathrm{d} \zeta=\mathrm{d} \vartheta, \zeta\left(t_{0}\right)=0 .
$$

By taking into account in the above formulas, the controls $\left(u_{i}, \vartheta_{i}\right)$ instead of $(u, \vartheta)$, for each $i$, we get functions $\alpha_{i}, \beta_{i}$ and, analogously, formulas for $\theta_{i}$ and $\operatorname{Ext}\left[\zeta_{i}, \vartheta_{i}\right]$, where $\theta_{i}$ is the inverse function associated with the impulsive control $\vartheta_{i}$.

Define $y_{i}:=\operatorname{Ext}\left[x_{i}, \vartheta_{i}\right]$. Then, we have

$$
\begin{aligned}
y_{i}(s)=x_{0, i} & +\int_{0}^{s} f\left(y_{i}(\varsigma), \operatorname{Ext}\left[u_{i}, \vartheta_{i}\right](\varsigma), \theta_{i}(\varsigma)\right) \alpha_{i}(\varsigma) \mathrm{d} \varsigma \\
& +\int_{0}^{s} g\left(y_{i}(\varsigma), \operatorname{Ext}\left[u_{i}, \vartheta_{i}\right](\varsigma), \theta_{i}(\varsigma)\right) \beta_{i}(\varsigma) \mathrm{d} \varsigma .
\end{aligned}
$$

By extracting a subsequence, in view of (3.2), and from the convergence $\left(u_{i}, \vartheta_{i}\right) \stackrel{\rho}{\rightarrow}(u, \vartheta)$, we deduce that $\theta_{i} \rightrightarrows \theta, \operatorname{Ext}\left[\zeta_{i}, \vartheta_{i}\right] \rightrightarrows \operatorname{Ext}[\zeta, \vartheta]$ and $\operatorname{Ext}\left[u_{i}, \vartheta_{i}\right](s) \rightarrow \operatorname{Ext}[u, \vartheta](s)$, a.a. $s \in[0,1]$, and also that $\alpha_{i}$ and $\beta_{i}$ are uniformly bounded. By passing to a subsequence once again and by using the weak sequential compactness of the unit ball in $\mathbb{L}_{2}$, we conclude that $\alpha_{i} \stackrel{*}{\rightarrow} \bar{\alpha}$, and $\beta_{i} \stackrel{*}{\rightarrow} \bar{\beta}$. After taking limits in

$$
\theta_{i}(s)=t_{0}+\int_{0}^{s} \alpha_{i}(\varsigma) \mathrm{d} \varsigma, \quad \operatorname{Ext}\left[\zeta_{i}, \vartheta_{i}\right](s)=\int_{0}^{s} \beta_{i}(\varsigma) \mathrm{d} \varsigma,
$$

we conclude that $\bar{\alpha}=\alpha$ and $\bar{\beta}=\beta$. 
From (4.6), bearing in mind (4.2) and by applying Gronwall's inequality, we conclude that functions $y_{i}$ are equicontinuous and uniformly bounded. By extracting a subsequence, and by using Arzela-Ascoli Theorem, we get $y_{i} \rightrightarrows y$. By passing to the limit as $i \rightarrow \infty$ in (4.6), we obtain

$$
\begin{aligned}
y(s)= & x_{0}+\int_{0}^{s} f(y(\varsigma), \operatorname{Ext}[u, \vartheta](\varsigma), \theta(\varsigma)) \alpha(\varsigma) \mathrm{d} \varsigma \\
& +\int_{0}^{s} g(y(\varsigma), \operatorname{Ext}[u, \vartheta](\varsigma), \theta(\varsigma)) \beta(\varsigma) \mathrm{d} \varsigma .
\end{aligned}
$$

But then, in view of the variable change, the trajectory $x(\cdot)=y(\pi(\cdot))$, where $\pi$, the inverse of $\theta$, satisfies (4.1). Hence, $y=\operatorname{Ext}[x, \vartheta]$. Since these arguments hold for any subsequence of the original sequence of functions $y_{i}(s)$, by the uniqueness of solution to differential equation, we have proved that $\operatorname{Ext}\left[x_{i}, \vartheta_{i}\right] \rightrightarrows \operatorname{Ext}[x, \vartheta]$.

The proof is complete.

\subsection{Approximation of impulsive controls}

The following lemma shows how, with the help of an integral functional, it becomes possible to approximate impulsive controls by conventional controls. Such lemma would be useful at proofs of various results in impulsive control theory and also for applications at theoretical justification of the convergence of approximating sequences (constructed via solving auxiliary linear-quadratic problems).

Lemma 4.3. Let $\left(\bar{u}_{i}, \bar{v}_{i}\right) \stackrel{\rho}{\rightarrow}(\bar{u}, \bar{\vartheta})$, and

$$
\int_{t_{0}}^{t_{1}}\left(\left|y_{i}(t)-\bar{y}_{i}(t)\right|^{2}+\left|u_{i}(t)-\bar{u}_{i}(t)\right|^{2}\right) m_{i}(t) \mathrm{d} t \rightarrow 0,
$$

where $y_{i}(t)=\int_{t_{0}}^{t}\left(v_{i},\left|v_{i}\right|\right) d \tau, \bar{y}_{i}(t)=\int_{t_{0}}^{t}\left(\bar{v}_{i},\left|\bar{v}_{i}\right|\right) d \tau$, and $m_{i}(t)=1+\frac{\left|v_{i}(t)\right|+\left|\bar{v}_{i}(t)\right|}{2}$, being the functions $u_{i}, \bar{u}_{i}$, $v_{i}$, and $\bar{v}_{i}$ of class $\mathbb{L}_{\infty}(T)$, and functions $u_{i}$ are uniformly bounded.

Then, $\left(u_{i}, v_{i}\right) \stackrel{\rho}{\rightarrow}(\bar{u}, \bar{\vartheta})$.

Proof can be found in [7]. This result, in some sense is connected to the approximation results in [33], where an impulsive dynamical system was investigated in the form of a measure-driven differential inclusion. The specific feature of the approach presented here is that the "impulsive control" information is accompanied with additional information about controls on the jumps, specifying the behavior of the dynamical system while the impulse develops. Such specification makes the state trajectory well and uniquely defined. According to this, the above approximation is made with respect to a specifically constructed metric $\rho$ that stores such impulse control information. In the work [33], a graph-convergence with respect to the Hausdorff metric was established. Thus, these results have different settings and do not really overlap.

\section{EXISTENCE THEOREMS}

In the previous sections a certain extension of control problems was defined for the case when admissible arcs enable discontinuities. Once the extension is defined, the main issue that remains is to understand whether and under which conditions such extension is successful in the sense that the solution to the extended problem does exist. Below, the existence under assumptions of Filippov's type is proved.

Hereinafter, by modulus of a vector we understand the sum of the absolute values of its coordinates, i.e., $|x|=\sum_{i}\left|x^{i}\right|$ and the unit sphere surface in $\mathbb{R}^{k}$, denoted by $S_{\mathbb{R}^{k}}$, is given by

$$
S_{\mathbb{R}^{k}}:=\left\{x \in \mathbb{R}^{k}:|x|=1\right\}
$$


Theorem 5.1. Assume that

(a) The sets $U$ and $S$ are compact.

(b) The set

$$
\bigcup_{\substack{u \in U, \alpha \in[0,1], v \in K \cap S_{\mathbb{R}^{k}}}} \alpha(f(x, u, t), 0,1)+(1-\alpha)(g(x, u, t) v, 1,0) \subseteq \mathbb{R}^{n+2}
$$

is convex for all $x, t$.

(c) The estimate (4.2) holds.

(d) The problem (2.1) has at least one admissible process.

Then, there exists a solution to problem (2.1).

Proof. Consider the auxiliary control problem

$$
\left\{\begin{array}{cl}
\text { minimize } & c(p) \\
\text { subject to } & \dot{x}=\alpha f(x, u, \chi)+(1-\alpha) g(x, u, \chi) v \\
& \dot{y}=1-\alpha, y_{0}=0 \\
& \dot{\chi}=\alpha, s \in\left[0, s_{1}\right] \\
& p=\left(x_{0}, x_{1}, y_{1}\right) \in S \\
& \chi_{0}=t_{0}, \chi_{1}=t_{1} \\
& \varphi(x, \chi) \leq 0 \\
& \alpha \in[0,1], v \in K,|v|=1
\end{array}\right.
$$

Unlike problem (2.1) considered on the fixed time interval $\left[t_{0}, t_{1}\right]$, problem (5.1) is considered on the nonfixed time interval $\left[0, s_{1}\right]$. Problem (5.1) possesses three control functions $u(s), v(s)$, and $\alpha(s)$, which are of conventional type, i.e., they are measurable and essentially bounded. Thereby, problem (5.1) is a conventional autonomous free time optimal control problem.

Let us show that the two problems, (2.1) and (5.1), are equivalent in the sense that, for every admissible process $(x, y, u, \vartheta)$ of problem (2.1), there exists an admissible process ( $\left.\tilde{x}, \tilde{y}, \chi, \tilde{u}, v, \alpha, s_{1}\right)$ of problem (5.1), such that $c(p)=c(\tilde{p})$, where $p=\left(x_{0}, x_{1}, y_{1}\right)$ and $\tilde{p}=\left(\tilde{x}_{0}, \tilde{x}_{1}, \tilde{y}_{1}\right)$, and vice versa.

First, let us be given an admissible process $(x, y, u, \vartheta)$ of problem (2.1). Consider the discontinuous time variable change

$$
\pi(t)=t-t_{0}+|\vartheta|\left(\left[t_{0}, t\right]\right) \text { as } t>t_{0}, \pi\left(t_{0}\right)=0 .
$$

Note that function $\pi(t)$ maps the interval $T$ into $\left[0, s_{1}\right]$, where

$$
s_{1}=t_{1}-t_{0}+|\vartheta|(T) \text {. }
$$

Let $\theta(s):\left[0, s_{1}\right] \rightarrow T$ be the inverse function of $\pi$.

By changing values of the function $u(t)$ on a zero $\ell$-measure set, we ensure that $u(t)$ is measurable w.r.t. the measure $\ell+|\vartheta|$. Indeed, it is enough to put $u(t)=0 \forall t \in D$, where $D$ is an arbitrary set of points of zero measure that the singular component of the measure $|\hat{\vartheta}|$ maps onto a full measure set. On the other hand, since $\ell(D)=0$, the values of $u$ do not affect the evolution of the trajectory, and, hence, this change of values of the function $u(\cdot)$ is not restrictive.

Let us take $\tilde{u}(s)=\operatorname{Ext}[u, \vartheta](s)$,

$$
\begin{aligned}
& \alpha(s)= \begin{cases}m_{1}(\theta(s)), \text { if } s \notin \bigcup_{\tau \in \operatorname{Ds}(\vartheta)} \Gamma_{\tau}, \\
0, \text { otherwise, }\end{cases} \\
& v(s)= \begin{cases}m_{2}(\theta(s)) /(1-\alpha(s)), & \text { if } s \notin \bigcup_{\tau \in \operatorname{Ds}(\vartheta)} \Gamma_{\tau} \text { and } \alpha(s)<1, \\
\left(\ell\left(\Gamma_{\tau}\right)\right)^{-1} v_{\tau}\left(\xi_{\tau}(s)\right), & \text { if } s \in \Gamma_{\tau},\end{cases}
\end{aligned}
$$


where, $m_{1}$ and $m_{2}$ are the Radon-Nykodym derivatives of the measures $\ell$ and $\mu_{c}$ with respect to $\ell+|\vartheta|$, $\xi_{\tau}(s):=\frac{s-\pi\left(\tau^{-}\right)}{\ell\left(\Gamma_{\tau}\right)}: \Gamma_{\tau} \rightarrow[0,1]$. When $\alpha(s)=1$, the values of $v(s)$ may be taken as arbitrary unit vectors from $K$, since the trajectory $x(\cdot)$ does not depend on those values. Note that $v(s) \in K$ and $|v(s)|=1$ due to $m_{1}(t)+\left|m_{2}(t)\right|=1$ a.a. $t \notin \operatorname{Ds}(\vartheta)$ w.r.t. the measure $\ell+|\vartheta|$.

By definition, it follows that $\theta(s)=t_{0}+\int_{0}^{s} \alpha(\varsigma) \mathrm{d} \varsigma$. Therefore, $\chi(s)=\theta(s)$. By performing the time variable change in $(2.2)$, it is easy to see that the trajectory $\tilde{x}(\cdot)$, solution to the dynamical system (5.1) corresponding to the just constructed collection $\left(x_{0}, \tilde{u}, v, \alpha, s_{1}\right)$, exists and it is exactly the function $\operatorname{Ext}[x, \vartheta](s)$. Thus, $\tilde{x}\left(s_{1}\right)=$ $x\left(t_{1}\right)$.

By construction, we also have that $\tilde{y}\left(s_{1}\right)=|\vartheta|(T)=y\left(t_{1}\right)$.

Therefore, $c(p)=c(\tilde{p})$ and $\tilde{p} \in S$. Moreover, $\varphi(\tilde{x}(s), \chi(s))=\varphi(\operatorname{Ext}[x, \vartheta](s), \theta(s)) \leq 0$ in view of definition of the state constraints.

So, the process constructed above is admissible to problem (5.1) and the cost function takes the same value, $c(p)$.

Conversely, let us be given an admissible process $\left(\tilde{x}, \tilde{y}, \chi, \tilde{u}, v, \alpha, s_{1}\right)$ of problem (5.1). The function $\chi(s)$ is the inverse of some discontinuous time variable change $\tilde{\pi}: T \rightarrow\left[0, s_{1}\right]$. The function $\tilde{\pi}$ is uniquely defined as the one that satisfies $\tilde{\pi}(\chi(s))=s$, a.a. $s$ such that $\alpha(s)>0, \tilde{\pi}\left(t_{0}\right)=0, \tilde{\pi}\left(t_{1}\right)=s_{1}$, and $\tilde{\pi}(t)$ is right-continuous in $\left(t_{0}, t_{1}\right)$. Once $\tilde{\pi}$ is determined, we find the measure $\mu$ via its distribution function

$$
F(t, \mu)=\int_{0}^{\tilde{\pi}(t)}(1-\alpha(s)) v(s) \mathrm{d} s .
$$

Let us take $u(t)=\tilde{u}(\tilde{\pi}(t)), u_{\tau}(s)=\tilde{u}\left(\tilde{\gamma}_{\tau}(s)\right), v_{\tau}(s)=\ell\left(\tilde{\Gamma}_{\tau}\right) v\left(\tilde{\gamma}_{\tau}(s)\right)$, where

$$
\begin{aligned}
\tilde{\Gamma}_{\tau} & =\left[\tilde{\pi}\left(\tau^{-}\right), \tilde{\pi}\left(\tau^{+}\right)\right], \\
\tilde{\gamma}_{\tau}(s) & =\ell\left(\tilde{\Gamma}_{\tau}\right) s+\tilde{\pi}\left(\tau^{-}\right):[0,1] \rightarrow \tilde{\Gamma}_{\tau} .
\end{aligned}
$$

Let $x(\cdot)$ be the solution defined by $(2.2)$. It follows right from the change of variable that $x\left(t_{1}\right)=\tilde{x}\left(s_{1}\right)$, and $y\left(t_{1}\right)=\tilde{y}\left(s_{1}\right)$. Clearly, the endpoint and the state constraints are satisfied. So, the constructed process is admissible to the problem (2.1), whereas the cost function takes the same value $c(\tilde{p})$.

Thus, we have shown that the two problems (2.1) and (5.1), are equivalent. Therefore, if a solution exists to one of the problems, then it also exists to the other.

Let us ensure the existence of solution to the auxiliary problem (5.1). The velocity set in (5.1) is convex due to the condition (b). This, together with other conditions (a), (c), (d), allows us to apply the classic Filippov's existence theorem to the problem (5.1). Thus, the solution to the auxiliary problem exists, and, hence, it exists to the original problem (2.1).

Above, it was implicitly used the fact that the endpoint constraints in (5.1) and the assumption (a) imply $s_{1} \leq$ const. Indeed, for the trajectory $y$ in (5.1), we have

$$
y\left(s_{1}\right)=\int_{0}^{s_{1}}(1-\alpha) \mathrm{d} s=s_{1}-\left(t_{1}-t_{0}\right) \stackrel{\text { a) }}{\Longrightarrow} s_{1} \leq \text { const } .
$$

The assumption (d) was used implicitly while performing the discontinuous variable change.

The proof is complete.

Condition (b) can be simplified when function $g$ does not depend on $u$, i.e., $g=g(x, t)$. Then, this condition is equivalent to convexity of the separate sets $f(x, U(x, t), t)$ and $g(x, t)\left(K \cap S_{\mathbb{R}^{k}}\right)$. In the general case, condition (b) implies separate convexity of the sets but not vice versa. Thereby, (b) is a stronger requirement than convexity of the separate velocity sets.

If the condition (b) is violated then the assertion of Theorem 5.1 is wrong, which can be confirmed by simple examples (when $g \equiv 0$ ), see [20]. 
Note that the condition (b) often implies that the intersection of the cone $K$ with the unit sphere should be convex. This is a fairly stringent requirement, since it immediately implies that the cone $K$ is embedded in one of the orthants in $\mathbb{R}^{k}$. However, when the above mentioned intersection is nonconvex, for example, if $K=\mathbb{R}^{k}$, $g=1$, the conclusions of Theorem 5.1 does not hold. Here is the corresponding counterexample.

Example 5.2. Consider the problem

$$
\left\{\begin{array}{cl}
\text { minimize } & \int_{0}^{1} x^{2} \mathrm{~d} t \\
\text { subject to } & \dot{x}=v \\
& \dot{y}=|v| \\
& x(0)=0, x(1)=0 \\
& y(0)=0, y(1)=1 \\
& v \in \mathbb{R}^{1} .
\end{array}\right.
$$

To see that the minimum of this problem is not achieved, consider the minimizing sequence of admissible trajectories

$$
x_{i}(t)=\left\{\begin{array}{ll}
t-\frac{j}{i}, & \text { if } t \in\left[\frac{j}{i}, \frac{2 j+1}{2 i}\right], \\
-t+\frac{j+1}{i}, & \text { if } t \in\left(\frac{2 j+1}{2 i}, \frac{j+1}{i}\right],
\end{array} \quad j=0,1, \ldots, i-1,\right.
$$

which converges uniformly to zero, but $\left.\operatorname{Var}\right|_{0} ^{1} x_{i}=1 \forall i$.

Then, there is a natural question of strengthening the assumptions in Theorem 5.1 in order to include the case $K=\mathbb{R}^{k}$, as well. The following theorem provides an answer to this question.

Theorem 5.3. Assume that:

(a) The set $U$ is compact and there are representations

- $S=C \times[0, a]$, where $C \subset \mathbb{R}^{2 n}$ is a compact set, and $a \in \mathbb{R}^{1}$ is a number, and

$-c(p)=c_{1}\left(x_{0}, x_{1}\right)+c_{2}\left(y_{1}\right)$, where $c_{1}$, and $c_{2}$ continuous functions such that $c_{2}$ is increasing.

(b) The set

$$
\bigcup_{\substack{u \in U, \alpha \in[0,1], v \in K \cap B_{\mathbb{R}^{k}}}} \alpha(f(x, u, t), 0,1)+(1-\alpha)(g(x, u, t) v, 1,0) \subseteq \mathbb{R}^{n+2}
$$

is convex for all $x, t$.

Suppose also that the assumptions (c) and (d) of Theorem 5.1 are satisfied.

Then, there exists a solution to the problem (2.1).

Proof. Consider the auxiliary problem

$$
\left\{\begin{array}{cl}
\text { Minimize } & c(p) \\
\text { subject to } & \dot{x}=\alpha f(x, u, \chi)+(1-\alpha) g(x, u, \chi) v \\
& \dot{y}=1-\alpha, y_{0}=0 \\
& \dot{\chi}=\alpha, s \in\left[0, s_{1}\right] \\
& p=\left(x_{0}, x_{1}, y_{1}\right) \in S \\
& \chi_{0}=t_{0}, \chi_{1}=t_{1} \\
& \varphi(x, \chi) \leq 0 \\
& \alpha \in[0,1], v \in K,|v| \leq 1
\end{array}\right.
$$

This problem is very similar to problem (5.1) but the constraint $|v|=1$ is replaced by $|v| \leq 1$. Let us show that if a solution exists for (2.1), then it also exists for (5.2), and vice versa. Note that, for every admissible process 


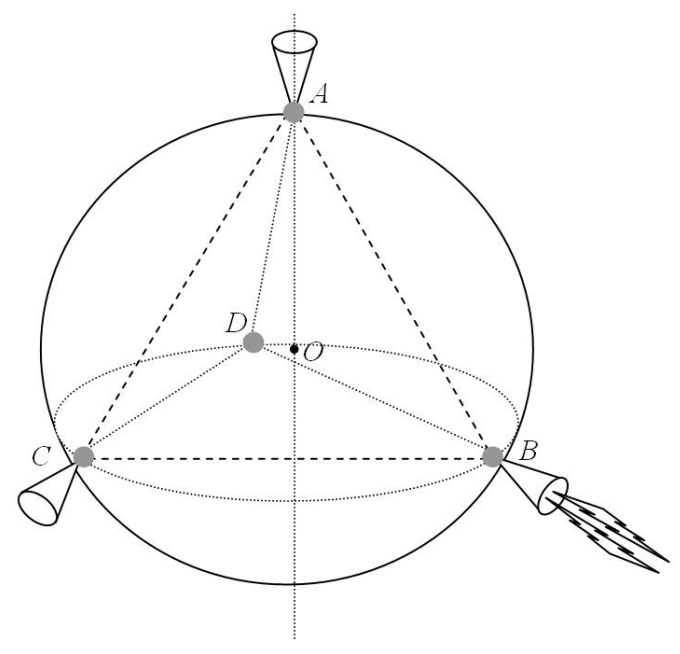

Figure 3. Four thrusters.

$(x, y, u, \vartheta)$ of problem $(2.1)$, there exists an admissible process $\left(\tilde{x}, \tilde{y}, \chi, \tilde{u}, v, \alpha, s_{1}\right)$ of the problem (5.2) such that $c(p)=c(\tilde{p})$, which is established by means of the same arguments as in the proof of Theorem 5.1.

Let $\left(\tilde{x}, \tilde{y}, \chi, \tilde{u}, v, \alpha, s_{1}\right)$ be an admissible process of the problem (5.2). Define an admissible process $(x, y, u, \vartheta)$ of $(2.1)$ such that $c(p) \leq c(\tilde{p})$. The objects $x, u, \vartheta$ are defined just by the same formulas as in the proof of Theorem 5.1, whereas $y(t)$ is taken as follows:

$$
y(t)=\int_{0}^{\tilde{\pi}(t)}(1-\alpha(s))|v(s)| \mathrm{d} s=\int_{\left[t_{0}, t\right]} \mathrm{d}|\vartheta|, \quad t>t_{0} .
$$

Since $S=C \times[0, a]$ in view of assumption (a), in view of $y_{1}\left(t_{1}\right) \leq \tilde{y}_{1}\left(s_{1}\right)$, the process $(x, y, u, \vartheta)$ is admissible in the original problem (2.1). Moreover, $c(p) \leq c(\tilde{p})$ by means of (a).

The above established facts imply that if a solution exists to one problem then it also exists to the other. However the solution to (5.2) exists by Filippov's Theorem which is applicable in view of (a), (b), (c), and (d).

The proof is complete.

Note 1. As an example of the function $c(\cdot)$ could serve any increasing function, e.g. $\ln \left(1+y_{1}\right)$ or $\exp \left(y_{1}\right)$. But the most natural and important case for applications is when the total variation is minimized, i.e., $c_{2}\left(y_{1}\right)=y_{1}$. This case corresponds to the functional of the model problem from Section 6, where the total variation of impulsive control will mean the fuel consumed during the flight.

\section{MOdel EXAMPLE}

The example below is a 3D development of the example from [9].

Consider a ball in the space $\mathbb{R}^{3}$ that can be maneuvered by the four thrusters located at points $A, B, C$, and $D$ as shown in Figure 3. Geometrically, $A B C D$ is a regular tetrahedron inscribed in the sphere. Assign numbers $1,2,3$ and 4 to the thrusters at points $A, B, C$ and $D$, respectively. Denote the center of the ball by $O$. For the center of gravity $G$ we assume that

$$
G \in \operatorname{int} A B C D \text {. }
$$

Consider a coordinate system with the origin in the center $O$ and axes as in Figure 4. The axis are chosen such that point $A$ belongs to $O z$ and the projection of point $B$ onto plane $O x y$ belongs to $O x$. It is assumed 


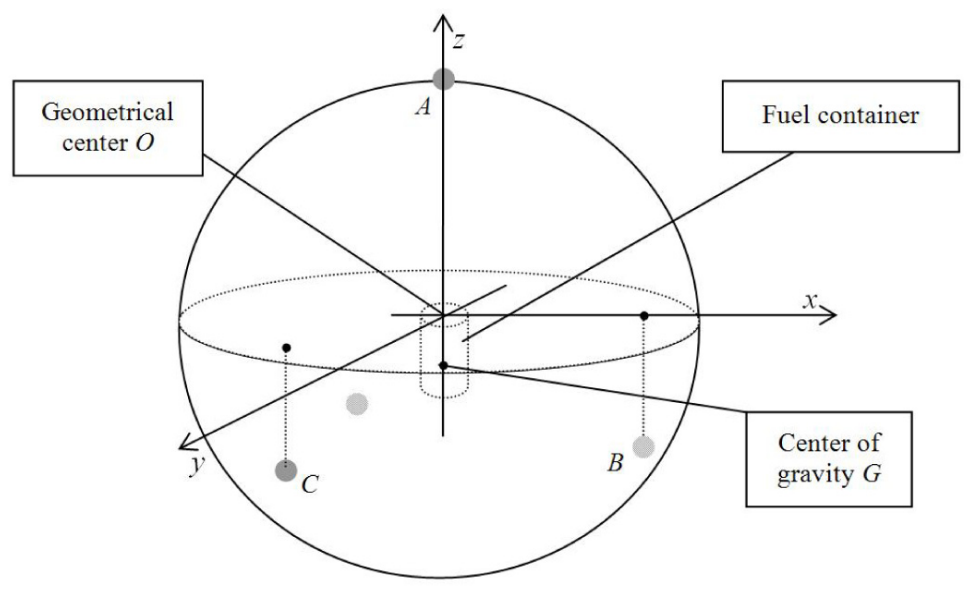

Figure 4. Coordinate system $O x y z$ and the fuel container.

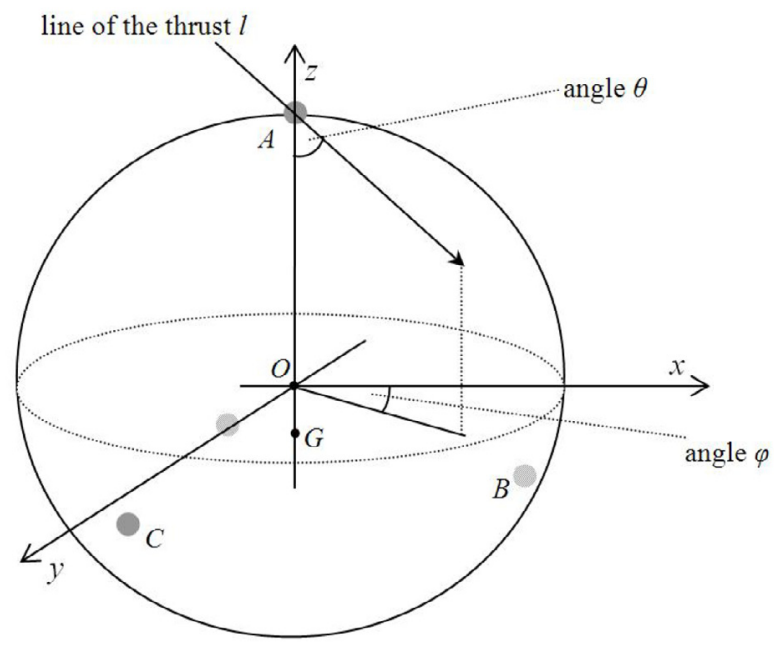

Figure 5. Angles $\theta$ and $\varphi$.

that the ball has a fuel container (see Fig. 4). The center of gravity location $G$ is a function of the fuel margin: $G:=G(m)$. When the container is empty, the center of gravity location coincides with the center of the ball $G=O$. But, when the fuel is present, point $G$ slides down by the $O z$ axis. The law of the center of gravity alteration is not specified precisely, but, in general, it can be any smooth function depending on $m$ with the values in the interior of $A B C D$ (not necessary concentrated in axis $O z$ ).

For each thruster, the controls are the direction $l_{i}$ of the propulsion force that is given by angles $\theta_{i}, \varphi_{i}$, $i=1, \ldots, 4$ (thruster inclination, see Fig. 5) and also the power coefficient $p_{i}, i=1, \ldots, 4$. To be more precise, for thruster number 1 , angles $\theta_{1}$ and $\varphi_{1}$ are exactly as in Figure 5, that is, $\theta_{1}=\angle O A l_{1}, \varphi_{1}=\angle x O l_{1}^{\prime}$, where the sign prime' means the projection onto plane $O x y$. For thruster number 2 , we have that $\theta_{2}=\angle G B l_{2}$, and angle $\varphi_{2}$ is specified similarly by the projection of line $l_{2}$ onto plane $\{B G\}^{\perp}$ in which axes are chosen in some fixed way. (For example, in such a way that axis $x$ is collinear to the projection of vector $G O$ onto $\{B G\}^{\perp}$ and 
the orientation of axes is counter clockwise with respect to vector $B G$ ). Angles $\theta_{3}, \varphi_{3}$ and $\theta_{4}, \varphi_{4}$ are specified in the same way as for the thruster at point $B$.

The vector of the propulsion force generated by each thruster has to be directed inside of or, at least, to be tangent to the sphere. Therefore, for example, for the thruster at point $A$ it should hold that $0 \leq \theta_{1} \leq \pi / 2$, $0 \leq \varphi_{1} \leq 2 \pi$. However, it is easy to see that already for the thruster at point $B$ angle $\theta_{2}$ cannot be $\pi / 2$ for every $\varphi_{2}$. Indeed, otherwise for some $\varphi_{2}$ the propulsion line $l_{2}$ is directed out of the sphere. Then, obviously, angle $\theta_{2}$ has to be restricted by the angle $\varphi_{2}$ and this restriction should also depend on mass $m$ since the center of gravity location, the point $G$, depends on $m$. Thus, the constraints

$$
0 \leq \theta_{2} \leq f_{2}\left(m, \varphi_{2}\right), \quad 0 \leq \varphi_{2} \leq 2 \pi
$$

where $f_{2}$ is some smooth function, appear. It is not specified, but finding $f_{2}$ is a straightforward stereometric task. Generally, by setting $f_{1}=\pi / 2$, the constraints for the control angles are

$$
0 \leq \theta_{i} \leq f_{i}\left(m, \varphi_{i}\right), \quad 0 \leq \varphi_{i} \leq 2 \pi, \quad i=1, \ldots, 4
$$

Constraints $\theta_{i} \leq f_{i}\left(m, \varphi_{i}\right)$ are called mixed because they involve state coordinate (mass) and controls. Note that the thrusters work in the orientation mode (with the zero translation) always corresponds to the angle value $\theta_{i}=\pi / 2$. However, this "pure orientation" mode is not always achievable for any direction of the angular velocity due to the technological restrictions induced by the mixed constraints.

In what concerns the power coefficients $p_{i}$, they should be nonnegative and should satisfy the norm equation

$$
p_{1}+p_{2}+p_{3}+p_{4}=1
$$

As it was mentioned above, the translation thrusters must satisfy the restriction of the force line intersecting the center of gravity (see Fig. 2). This guarantees zero rotation (no fuel spent for rotation). Such driving mode has a low control authority, as it does not allow us to reorient the ball. In the case when it is necessary to reorient and to translate the ball at the same time, the force line does not necessarily intersect the center of gravity. Thus, it can be decomposed in two orthogonal components one of which passes through the center of gravity and responds for translation, and the other one responds for rotation.

The first aim now is to find equations of translation and rotation motions. Let $e_{i}(m)$ be unit vectors directed from the thrusters to the center of gravity, i.e. for instance $e_{2}(m)=B G /|B G|$. These vectors are defined in coordinate system $O x y z$ related with the ball (that is in the rotating system). However, in order to write the equations of translation motion, we also need to use a coordinate system that is not related with the ball and in which the ball is a particle. Consider such a fixed system $O x_{1} x_{2} x_{3}$. Let $\alpha$ denote the vector angle specified by the orientation of the ball in system $O x_{1} x_{2} x_{3}$. More precisely, the vector $\alpha$ has three components $-\alpha_{23}, \alpha_{13}, \alpha_{12}$ - which are the rotation angles about the axes $O+0 x_{1}, O+0 x_{2}$, and $O+0 x_{3}$ in the planes $O+0 x_{2} x_{3}$, $O+0 x_{1} x_{3}$, and $O+0 x_{1} x_{2}$ respectively. Note that $\alpha=0$ if $O x y z=O+0 x_{1} x_{2} x_{3}$. The positive rotations are counter-clockwise.

Let $T(\alpha)$ be the following matrix of rotation by $\alpha$

$$
T(\alpha)=\left[\begin{array}{ccc}
\cos \alpha_{12} & -\sin \alpha_{12} & 0 \\
\sin \alpha_{12} & \cos \alpha_{12} & 0 \\
0 & 0 & 1
\end{array}\right] \times\left[\begin{array}{ccc}
\cos \alpha_{13} & 0 & -\sin \alpha_{13} \\
0 & 1 & 0 \\
\sin \alpha_{13} & 0 & \cos \alpha_{13}
\end{array}\right] \times\left[\begin{array}{ccc}
1 & 0 & 0 \\
0 & \cos \alpha_{23} & -\sin \alpha_{23} \\
0 & \sin \alpha_{23} & \cos \alpha_{23}
\end{array}\right]
$$

Note that $O x y z=O+T(\alpha) 0 x_{1} x_{2} x_{3}$. 
Now the equations of the translation motion are:

$$
\begin{aligned}
& \dot{x}=w, \\
& \dot{w}=g(x)+T(\alpha) \sum_{i=1}^{4} p_{i} \cos \theta_{i} e_{i}(m) \cdot \frac{v}{m}, \\
& \dot{m}=-v .
\end{aligned}
$$

Here,

- $x=\left(x_{1}, x_{2}, x_{3}\right)$ is the particle position;

- $w=\left(w_{1}, w_{2}, w_{3}\right)$ is the particle velocity;

- $m$ is the particle mass. The mass of the ball is not constant and it changes in time while the fuel is consumed;

- $v$ is the fuel consumption rate which is nonnegative scalar; and

- $g(x)$ is the vector function specifying the gravitational field, thus defining the gravity acceleration at point $x$ of the space.

Above, the absolute value of the propulsion force by the thruster is proportional to its fuel consumption rate.

For thruster $i$, in the coordinate system $O x y z$, consider the rotation matrix $U_{i}(m)$ that maps plane $O x y$ into the corresponding thruster tangent plane in such a way that vector $U_{i}(m) \times\left(\cos \varphi_{i}, \sin \varphi_{i}, 0\right)^{\top}$ is collinear with the orientation component of the thrust. In other words, $U_{1}(m)=E$, where $E$ is identity matrix, $U_{2}(m)$ maps $O x y$ onto $\{B G\}^{\perp}$ with the required axes orientation and so on. Let us put

$$
d_{i}\left(m, \varphi_{i}\right)=U_{i}(m) \times\left(\cos \varphi_{i}, \sin \varphi_{i}, 0\right)^{\top} .
$$

Denote the distance between thruster $i$ and point $G$ by $r_{i}(m)$; the moment of inertia matrix about the axes $G+0 x_{1}, G+0 x_{2}, G+0 x_{3}$ by $I(m)$; and the angular velocity with respect to the center of gravity by $\omega$.

The sum of the torques by the forces is

$$
\tau=T(\alpha) \sum_{i=1}^{4} p_{i} r_{i}(m) \sin \theta_{i}\left[d_{i}\left(m, \varphi_{i}\right) \times e_{i}(m)\right] \cdot v,
$$

where denotation $[a \times b]$ is used for the vector product of $a$ and $b$.

For the differential of the angular momentum, we have

$$
\mathrm{d} L=I(m+\mathrm{d} m)(\omega+\mathrm{d} \omega)-\sum_{i=1}^{4} p_{i} r_{i}^{2}(m) \omega \mathrm{d} m-I(m) \omega,
$$

where, in computing the angular momentum of the consumed fuel, it was also used the fact that, for two orthogonal vectors $a$ and $b$, it holds that $a=[b \times[a \times b]]$. By conservation of angular momentum it follows that $\mathrm{d} L=\tau \mathrm{d} t$. Then, by using that $\dot{m}=-v$, the following equation for the orientation angle $\alpha$ is obtained

$$
\begin{aligned}
& \dot{\alpha}=\omega, \\
& \dot{\omega}=I^{-1}(m)\left(I^{\prime}(m) \omega-\sum_{i=1}^{4} p_{i} r_{i}^{2}(m) \omega+T(\alpha) \sum_{i=1}^{4} p_{i} r_{i}(m) \sin \theta_{i}\left[d_{i}\left(m, \varphi_{i}\right) \times e_{i}(m)\right]\right) \cdot v,
\end{aligned}
$$

where $I^{\prime}(m)=\mathrm{d} I / \mathrm{d} m$ is the derivative of the moment of inertia matrix with respect to mass. 
Now, by means of the equations of motion derived in the previous section, the optimal fuel consumption problem associated with the motion of the ball from point $A$ to point $B$ takes the following form:

$$
\begin{cases}\text { minimize } & m(0) \\ \text { subject to } \dot{x}=w, \quad x(0)=A, x(1)=B, \\ & \dot{w}=g(x)+T(\alpha) \sum_{i=1}^{4} p_{i} \cos \theta_{i} e_{i}(m) \cdot \frac{v}{m}, \quad w(0)=w_{A}, w(1)=w_{B}, \\ & \dot{m}=-v, \quad m(1)=M, \\ & \dot{\alpha}=\omega, \quad \alpha(0)=\alpha_{A}, \alpha(1)=\alpha_{B}, \\ \dot{\omega}=I^{-1}(m)\left(I^{\prime}(m) \omega-\sum_{i=1}^{4} p_{i} r_{i}^{2}(m) \omega+T(\alpha) \sum_{i=1}^{4} p_{i} r_{i}(m) \sin \theta_{i}\left[d_{i}\left(m, \varphi_{i}\right) \times e_{i}(m)\right]\right) \cdot v & \omega(0)=\omega_{A}, \omega(1)=\omega_{B}, \\ & 0 \theta_{i} \leq f_{i}\left(m, \varphi_{i}\right), \quad 0 \leq \varphi_{i} \leq 2 \pi, \quad i=1, \ldots, 4, \\ & \sum_{i=1}^{4} p_{i}=1, p_{i} \geq 0, v \geq 0,\end{cases}
$$

where $M$ is the mass of the ball without fuel.

Note, the control $v$ is assumed to be unbounded. Therefore, we expect that problem (6.1) has no solution in the class of ordinary bounded controls. Then, the solution is found in the class of impulsive controls.

By setting the problem (6.1) in the impulsive framework as stated in Section 2, we obtain the following impulsive control problem

$$
\begin{cases}\text { minimize } & m(0) \\ \text { subject to } & \dot{x}=w, \quad x(0)=A, x(1)=B, \\ & d w=g(x)+T(\alpha) \frac{\sum_{i=1}^{4} p_{i} \cos \theta_{i} e_{i}(m)}{m} \mathrm{~d} \vartheta, \quad w(0)=w_{A}, \quad w(1)=w_{B}, \\ & \mathrm{~d} m=-\mathrm{d} \vartheta, \quad m(1)=M, \\ & \dot{\alpha}=\omega, \quad \alpha(0)=\alpha_{A}, \alpha(1)=\alpha_{B}, \\ & \mathrm{~d} \omega=I^{-1}(m)\left(I^{\prime}(m) \omega-\sum_{i=1}^{4} p_{i} r_{i}^{2}(m) \omega+T(\alpha) \sum_{i=1}^{4} p_{i} r_{i}(m) \sin \theta_{i}\left[d_{i}\left(m, \varphi_{i}\right) \times e_{i}(m)\right]\right) \mathrm{d} \vartheta \\ & \\ 0 \leq \theta_{i} \leq f_{i}\left(m, \varphi_{i}\right), 0 \leq \varphi_{i} \leq 2 \pi, \quad i=1, \ldots, 4 & \\ & \sum_{i=1}^{4} p_{i}=1, p_{i} \geq 0, \\ & \vartheta=\left(\left.\left\{\left(\varphi_{i, \tau}, \theta_{i, \tau}, p_{i, \tau}\right)\right\}\right|_{\tau \in[0,1], i=1, \ldots, 4}, \mu\right), \mu \geq 0 .\end{cases}
$$

Here, $\vartheta=\left(\left.\left\{\left(\varphi_{i, \tau}, \theta_{i, \tau}, p_{i, \tau}\right)\right\}\right|_{\tau \in[0,1], i=1, \ldots, 4}, \mu\right)$ is the impulsive control. Linear and angular velocities and mass trajectories are now discontinuous functions. For example, a velocity jump at point $\tau$ such that $\mu(\{\tau\})>0$ is computed by means of adjoint controls and adjoint system on segment $[0,1]$ as follows:

$$
\begin{aligned}
& \dot{w}_{\tau}(s)=g(x(\tau))+T(\alpha(\tau)) \sum_{i=1}^{4} p_{i, \tau}(s) \cos \theta_{i, \tau}(s) e_{i}\left(m_{\tau}(s)\right) m_{\tau}(s)^{-1} \mu(\{\tau\}), \\
& \dot{m}_{\tau}(s)=-\mu(\{\tau\}),
\end{aligned}
$$

where $w_{\tau}(0)=w\left(\tau^{-}\right), m_{\tau}(0)=m\left(\tau^{-}\right), w_{\tau}(1)=w\left(\tau^{+}\right)$, and $m_{\tau}(1)=m\left(\tau^{+}\right)$, where $f\left(\tau^{-}\right), f\left(\tau^{+}\right)$denotes leftand right-hand limits of $f$ at point $\tau$.

Note that we must have $x_{3} \geq 0$, as this coordinate represents the altitude, in which $x_{3}=0$ is the ground level (e.g., the case of the lunar module at landing). Moreover, $|\omega| \leq$ const. because of the natural technological restriction. The centrifugal force would inevitably destroy the ball if $\omega$ were sufficiently large. Also, $|w| \leq$ const. is a naturally encountered restriction (air resistance, thermal barrier, etc.). This clearly illustrates how the state 
constraints of inequality type can be involved. Thus, the relevance of the problem statement from Section 2 has been shown.

Let us say a few words about the existence of impulsive minimizers to problem (6.2). Let $f_{i}$ 's be constant and, thus, the mixed constraints specified by $f_{i}$ and involved in the problem setting in (6.2) become conventional geometric constraints on $\theta_{i}$. Then, it is easy to check that the velocity set to the right-hand in (6.2) is convex and compact, and thus, the application of Theorem 5.1 directly implies the existence of solution. However, in the presence of the mixed constraints, the existence would hold only for concave in $\varphi_{i}$ functions $f_{i}$.

At this point, it is also important to mention the following. The well-posed problem formulation relatively to the so-called degeneracy phenomenon in optimal control problems with state constraints was given in [3] (first raised in [10], see also [6] for the impulsive control context). The problem statement of (6.2) naturally meets the controllability requirements imposed in $[3,6]$ and thereby the necessary optimality conditions become meaningful if the state constraints of the mentioned type are involved.

\section{Closing COnsiderations}

The research reported in this article is a part of a long term effort in extending results from well established control theory for conventional control systems to impulsive control framework. Here, the problem of properness of the extension was addressed including well-posedness of the solution to the impulsive control system in the Cauchy sense, the well-posedness of passing to the limit of sequences of control processes, and also existence theorems. This enables to investigate the convergence of approximating sequences of impulsive controls and attests the well-posedness of the solution concept earlier developed by the authors and adopted in this article. Moreover, the results of this article can be regarded as extensions of results and approaches considered earlier in $[22,26,27,29]$ to the new solution concept.

It has been shown how, with the help of only the Lebesgue discontinuous variable change and a certain reduction, it becomes possible to remove the problem of well-posedness of the impulsive Cauchy problem and also to prove Filippov's existence theorems, thereby establishing properness of the impulsive extension from Section 2.

The article ends up with a model example showing the relevance of impulsive controls in possible engineering applications. In particular it shows how some complex control processes involving rapid variations in mass distribution of a mechanical system may be modelled or described by the impulsive controls introduced in Section 2.

Acknowledgements. The first and fourth authors were supported by CNPq (Brazil) "Sem Fronteiras" grant number 401689/2012-3. The first author was also supported by the Russian Foundation for Basic Research, grant number 1301-00494. The third author was supported by FCT (Portugal) grants Incentivo/EEI/UI0147/2014, and FCT funded project PEst-OE/EEI/UI0147/2014. The second and fourth authors were supported by Sao Paulo Research Foundation (FAPESP - CEPID) grant number 2013/07375-0 and CNPq (Brazil) grant number 479109/2013-3. The fourth author was also supported by CNPq (Brazil) grant number 309335/2012-4.

We kindly thank the anonymous referees for their substantial help in improving the exposition of the results in this work.

\section{REFERENCES}

[1] M.A. Aronna and F. Rampazzo, A note on systems with ordinary and impulsive controls. IMA J. Math. Control Inf. (2014) DOI : 10.1093/imamci/dnu033.

[2] A.V. Arutyunov, Optimality Conditions: Abnormal and Degenerate Problems. Kluwer Academic Publishers, Dordrecht, Boston, London (2000).

[3] A.V. Arutyunov and S.M. Aseev, State constraints in optimal control. The degeneracy phenomenon. Systems Control Lett. 26 (1995) 267-273.

[4] A.V. Arutyunov, V. Dykhta and F.L. Pereira, Necessary conditions for impulsive nonlinear optimal control problems without a priori normality assumptions. J. Optimization Theory Appl. 124 (2005) 55-77. 
[5] A.V. Arutyunov, V. Jacimovic and F.L. Pereira, Second order necessary conditions for optimal impulsive control problems. J. Dynam. Control Syst. 9 (2003) 131-153.

[6] A.V. Arutyunov, D. Karamzin and F.L. Pereira, A nondegenerate maximum principle for the impulse control problem with state constraints. SIAM J. Control Optim. 43 (2005) 1812-1843.

[7] A.V. Arutyunov, D.Yu. Karamzin and F.L. Pereira, On impulsive control problems with constraints: control of jumps of systems. J. Math. Sci. 165 (2010) 654-688.

[8] A.V. Arutyunov, D.Yu. Karamzin and F.L. Pereira, On the extension of classical calculus of variations and optimal control to problems with discontinuous trajectories, in Proc. of the 51st IEEE Conference on Decision and Control, CDC 2012. Maui, Hawaii (2012) 6406-6411.

[9] A.V. Arutyunov, D.Yu. Karamzin and F.L. Pereira, Pontryagin's maximum principle for constrained impulsive control problems. Nonlin. Anal. 75 (2012) 1045-1057.

[10] A.V. Arutyunov and N.T. Tynyanskiy, The maximum principle in a problem with phase constraints. Soviet J. Comput. Systems Sci. 23 (1985) 28-35.

[11] A. Bressan and F. Rampazzo, Impulsive control systems with commutative vector fields. J. Optimization Theory Appl. 71 (1991) 67-83.

[12] A. Bressan and F. Rampazzo, On differential systems with vector-valued impulsive controls. Boll. Un. Mat. Ital. B 72 (1988) 641-656.

[13] W.J. Code and P.D. Loewen, Optimal control of non-convex Measure-driven differential inclusions. Set-Valued Var. Anal. 19 (2011) 203-235.

[14] W.J. Code and G.N. Silva, Closed loop stability of measure-driven impulsive control systems. J. Dyn. Control Syst. 16 (2010) $1-21$.

[15] V.A. Dykhta and O.N. Samsonyuk, Optimal Impulse Control with Applications. Fizmatlit, Moscow (2000) [in Russian].

[16] N. Forcadel, Z. Rao and H. Zidani, State-constrained optimal control problems of impulsive differential equations. Appl. Math. Optim. 68 (2013) 1-19.

[17] A.B. Kurzhanski and A.N. Daryin, Dynamic programming for impulse controls. Ann. Rev. Control 32 (2008) $213-227$.

[18] D.F. Lawden, Optimal Trajectories for Space Navigation. Butterworth, London (1963).

[19] B.M. Miller and E.Ya. Rubinovich, Impulsive Control in Continuous and Discrete-Continuous Systems. Kluwer Academic/Plenum Publishers, New York (2003).

[20] B. Mordukhovich, Existence of optimal controls. Itogi Nauki Tech. Sovr. Prob. Mat. 6 (1976) 207-261. (Russian); English transl. in J. Soviet Math. 7 (1977) 850-886.

[21] B. Mordukhovich, Variational Analysis and Generalized Differentiation. Springer-Verlag, Berlin (2006).

[22] F.L. Pereira and G.N. Silva, Necessary conditions of optimality for vector-valued impulsive control problems. Syst. Control Lett. 40 (2000) 205-215.

[23] F.L. Pereira and G.N. Silva, Stability for impulsive control systems. Dyn. Syst. 17 (2002) 421-434.

[24] F.L. Pereira, G.N. Silva and V.A. de Oliveira, Invariance for impulsive control systems. Autom. Remote Control 69 (2008) $788-800$.

[25] L.S. Pontryagin, V.G. Boltyanskii, R.V. Gamkrelidze and E.F. Mishchenko, Mathematical Theory Optimal Processes. Classics Sov. Math. Nauka, Moscow (1983).

[26] G.N. Silva and R.B. Vinter, Measure driven differential inclusions. J. Math. Anal. Appl. 202 (1996) 727-746.

[27] G.N. Silva and R.B. Vinter, Necessary conditions for optimal impulsive control problems. SIAM J. Control Optim. 35 (1997) 1829-1846.

[28] R.B. Vinter, Optimal Control. Birkhäuser, Boston (2000).

[29] R.B. Vinter and F.L. Pereira, A maximum principle for optimal processes with discontinuous trajectories. SIAM J. Control Optim. 26 (1988) 205-229.

[30] R.W. Rishel, An extended Pontryagin principle for control systems whose control laws contain measures. SIAM J. Control Ser. A 3 (1965) 191-205.

[31] J. Warga, Variational problems with unbounded controls. SIAM J. Control Ser. A 3 (1965) $424-438$.

[32] P.R. Wolenski and S. Zabic, A differential solution concept for impulsive systems. Dyn. Contin. Discrete Impuls. Syst., Ser. A Math. Anal. 13B (2006) 199-210.

[33] P.R. Wolenski and S. Zabic, A sampling method and approximation results for impulsive systems. SIAM J. Control Optim. 46 (2007) 83-998. 